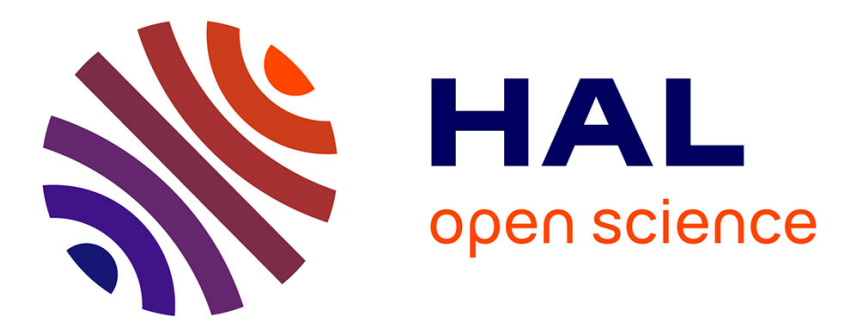

\title{
Antagonism between $\beta$-catenin and Gata.a sequentially segregates the germ layers of ascidian embryos
}

Kaoru S. Imai, Clare Hudson, Izumi Oda-Ishii, Hitoyoshi Yasuo, Yutaka Satou

\section{To cite this version:}

Kaoru S. Imai, Clare Hudson, Izumi Oda-Ishii, Hitoyoshi Yasuo, Yutaka Satou. Antagonism between $\beta$-catenin and Gata.a sequentially segregates the germ layers of ascidian embryos. Development (Cambridge, England), 2016, 143 (22), pp.4167 - 4172. 10.1242/dev.141481 . hal-01405916

\section{HAL Id: hal-01405916 https://hal.sorbonne-universite.fr/hal-01405916}

Submitted on 5 Dec 2016

HAL is a multi-disciplinary open access archive for the deposit and dissemination of scientific research documents, whether they are published or not. The documents may come from teaching and research institutions in France or abroad, or from public or private research centers.
L'archive ouverte pluridisciplinaire $\mathbf{H A L}$, est destinée au dépôt et à la diffusion de documents scientifiques de niveau recherche, publiés ou non, émanant des établissements d'enseignement et de recherche français ou étrangers, des laboratoires publics ou privés. 


\title{
Antagonism between $\beta$-catenin and Gata.a sequentially segregates the germ layers of ascidian embryos
}

\author{
Kaoru S. Imai ${ }^{1,2, *}$, Clare Hudson ${ }^{3, *}$, Izumi Oda-Ishii ${ }^{2, *}$, Hitoyoshi Yasuo ${ }^{3, \ddagger}$ and Yutaka Satou ${ }^{2, \ddagger}$
}

\begin{abstract}
Many animal embryos use nuclear $\beta$-catenin ( $n \beta$-catenin) during the segregation of endomesoderm (or endoderm) from ectoderm. This mechanism is thus likely to be evolutionarily ancient. In the ascidian embryo, $\mathrm{n} \beta$-catenin reiteratively drives binary fate decisions between ectoderm and endomesoderm at the 16-cell stage, and then between endoderm and margin (mesoderm and caudal neural) at the 32-cell stage. At the 16-cell stage, $\mathrm{n} \beta$-catenin activates endomesoderm genes in the vegetal hemisphere. At the same time, $n \beta$-catenin suppresses the DNA-binding activity of a maternal transcription factor, Gata.a, through a physical interaction, and Gata.a thereby activates its target genes only in the ectodermal lineage. In the present study, we found that this antagonism between $\mathrm{n} \beta$-catenin and Gata.a also operates during the binary fate switch at the 32-cell stage. Namely, in marginal cells where $n \beta$-catenin is absent, Gata.a directly activates its target, Zic-r.b (ZicL), to specify the marginal cell lineages. Thus, the antagonistic action between $n \beta$-catenin and Gata.a is involved in two consecutive stages of germ layer segregation in ascidian embryos.
\end{abstract}

KEY WORDS: $\beta$-Catenin, Ciona intestinalis, Gata, Zic, Ascidian, Germ layer formation

\section{INTRODUCTION}

In early embryos of many invertebrate animals, including echinoderms, hemichordates, ascidians, nematodes and cnidarians, nuclear localized $\beta$-catenin (n $\beta$-catenin) is used for the specification of endomesoderm (or endoderm) (Darras et al., 2011; Logan et al., 1999; Maduro, 2009; McCauley et al., 2015; Momose and Houliston, 2007; Wikramanayake et al., 2003, 1998). This process is thus likely to be an evolutionarily ancient mechanism.

In embryos of the chordate Ciona intestinalis, $\mathrm{n} \beta$-catenin is not only involved in the segregation of endomesoderm from ectoderm but also in the subsequent segregation of endoderm from mesoderm (and some neural) lineages (Hudson et al., 2013; Imai et al., 2000). Thus, germ layer segregation in Ciona embryos begins with two temporally separable steps.

In the first step, which occurs at the 16-cell stage, $n \beta$-catenin activity promotes endomesoderm over ectoderm lineage-specific

\footnotetext{
${ }^{1}$ Department of Biological Sciences, Graduate School of Science, Osaka University, Toyonaka, Osaka 560-0043, Japan. ${ }^{2}$ Department of Zoology, Graduate School of Science, Kyoto University, Kyoto 606-8502, Japan. ${ }^{3}$ Sorbonne Universités, UPMC Univ Paris 06, CNRS, Laboratoire de Biologie du Développement de Villefranche-sur-mer, Observatoire Océanologique, Villefranche-sur-mer 06230, France.

*These authors contributed equally to this work

${ }^{\ddagger}$ Authors for correspondence (yasuo@obs-vlfr.fr; yutaka@ascidian.zool.kyoto-u.ac.jp)

Y.S., 0000-0001-5193-0708
}

Received 22 June 2016; Accepted 22 September 2016 gene expression. In vegetal cells, from which endomesoderm is derived, Foxd and $F g f 9 / 16 / 20$ are directly activated by $n \beta$-catenin and its partner transcription factor Tcf7 (Imai et al., 2002a; OdaIshii et al., 2016). Conversely, Efna.d [formerly called EphrinA-d, and renamed according to the nomenclature guidelines for this animal (Stolfi et al., 2015)] and Tfap2-r.b are activated by the Gata.a transcription factor in animal cells, from which ectoderm is derived (Bertrand et al., 2003; Horikawa et al., 2013; Oda-Ishii et al., 2016; Rothbächer et al., 2007). Although Gata.a is present ubiquitously in the embryo, its activity is inhibited in vegetal cells by a physical interaction with $\beta$-catenin and Tcf7 (Oda-Ishii et al., 2016). This interaction thus restricts Gata.a activity to the animal hemisphere. Therefore, in vegetal cells, $\beta$-catenin/Tcf7 directly promotes the transcriptional activation of endomesoderm genes, and indirectly inhibits the expression of ectoderm genes. In the animal hemisphere, Gata.a, free from $\beta$-catenin/Tcf7-mediated repression, initiates the ectoderm-specific genetic program.

The second $n \beta$-catenin binary fate decision takes place at the 32cell stage, following a cell division that segregates the endoderm lineages (E cells) from the marginal (mesoderm and some neural) lineages (the NN and MM cells) (Fig. 1A). During this step, $n \beta-$ catenin promotes endoderm over marginal lineage specification, such that Zic-r.b (formerly ZicL) is specifically activated in margin cells, and $L h \times 3 / 4$ (formerly $L h \times 3$ ) is activated in E cells (Hudson et al., 2013; Imai et al., 2002b; Satou et al., 2001). Zic-r.b is a key specifier for the marginal lineages, required for the anterior marginal cells (NN cells, A6.2 and A6.4) to give rise to notochord and caudal neural tissue, and for each of the posterior marginal cells [anterior MM (aMM) cells, B6.2; posterior MM (pMM) cells, B6.4] to give rise to both muscle and mesenchyme (Imai et al., 2006, 2002b; Satou and Imai, 2015; Yagi et al., 2004). It appears that Zic-r.b is regulated differently between aMM and $\mathrm{pMM}$; when $\mathrm{n} \beta$-catenin is ectopically activated, Zic-r.b expression is lost in NN and aMM cells but not in pMM (Hudson et al., 2013). In the present study, we address how Zic-r.b is activated differentially between NN/aMM cells and E cells, and examine the possibility that the physical association of Gata.a and $\beta$-catenin/ Tcf7 might also be involved during the segregation of cell lineages at the 32-cell stage.

\section{RESULTS AND DISCUSSION}

\section{Gata.a is required for Zic-r.b expression}

In order to test whether Gata.a is required for Zic-r.b expression in marginal cells, we knocked down Gata.a using morpholino oligonucleotides (MOs). As shown in Fig. 1B, Zic-r.b was expressed normally in NN and MM cells in embryos injected with a control MO against Escherichia coli lacZ. By contrast, in embryos injected with Gata.a-MO, Zic-r.b expression was reduced in NN and aMM cells, but not in pMM cells (Fig. 1C,D; Fig. S1). Thus, Gata.a is required for Zic-r.b expression in NN and aMM cells, but is dispensable for expression in pMM cells. 
A

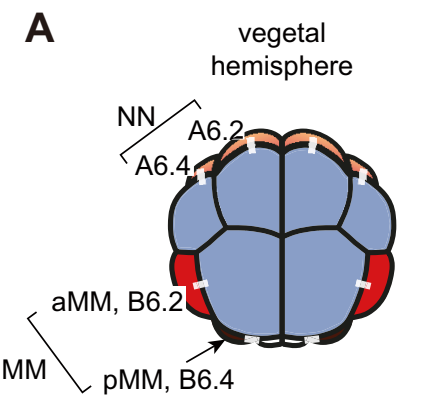

$$
\begin{aligned}
& \square \text { NN cell (Zic-r.b; } \\
& \text { Notochord \& Nerve cord) }
\end{aligned}
$$

aMM cell (Zic-r.b;

Muscle, Mesenchyme \& Notochord)

$$
\text { pMM cell (Zic-r.b; }
$$
Muscle \& Mesenchyme)

\section{$\square$ E cell (Lhx3/4; Endoderm)}

B
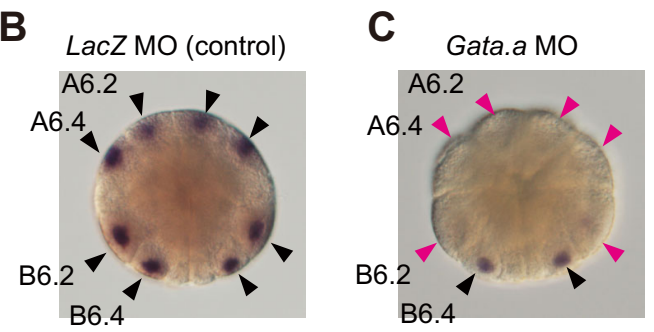

D $(\%)$

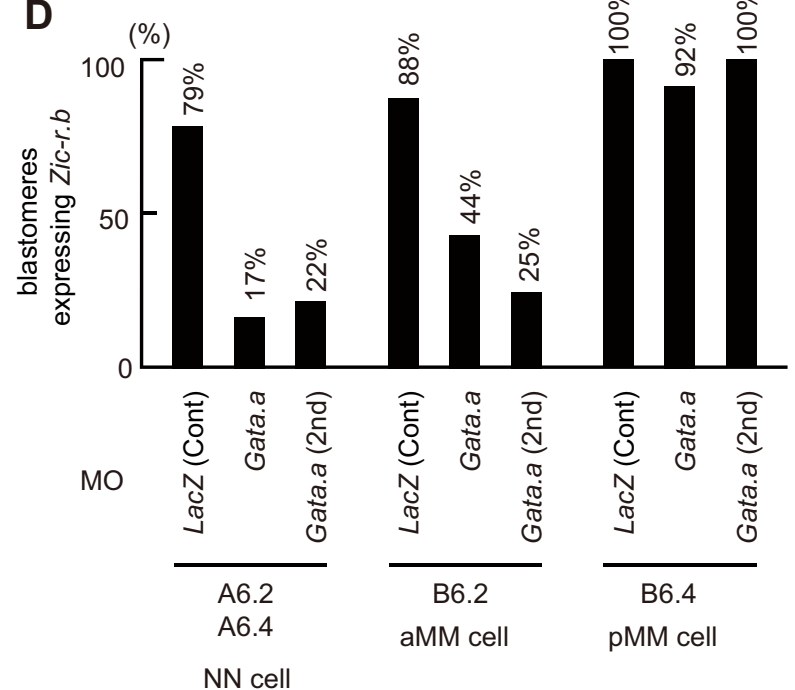

Fig. 1. Zic-r.b expression in the marginal cells is regulated by Gata.a. (A) An illustration of the 32-cell embryo. Because the embryo is bilaterally symmetrical, blastomere names are shown only in the left half. At this stage, the developmental fate of the animal hemisphere cells (yellow) is restricted to ectoderm, and the developmental fate of the vegetal-most cells (blue) is restricted to mostly endoderm. The marginal cells, NN and MM cells, with distinct lineage origins express Zic-r.b and give rise to mesodermal tissues and posterior neural tube. White bars connecting two cells indicate their sister cell relationship. (B-D) Zic-r.b expression in embryos injected with a lacZ control $\mathrm{MO}(\mathrm{B})$ and a Gata.a MO (C). Unfertilized eggs were injected with either of the MOs $(1 \mathrm{mM})$, and inseminated $1 \mathrm{~h}$ after injection. Black arrowheads indicate Zic-r.b expression and magenta arrowheads indicate loss of Zic-r.b expression. We analyzed 40 lacZ morphants and 31 Gata.a morphants. We also analyzed 16 embryos injected with a second MO against Gata.a. The percentages of blastomeres that expressed Zic-r.b are shown in D.

\section{Gata.a directly activates Zic-r.b expression}

Zic-r.b is a multi-copy gene (Dehal et al., 2002; Yamada et al., 2003). By comparing the upstream regulatory regions of four copies, we found that the region approximately $230 \mathrm{bp}$ upstream from the transcription start sites was highly conserved (Fig. S2A). We identified four putative Gata-binding sites in this highly conserved domain. Chromatin-immunoprecipitation (ChIP) mapping of Gata.a binding, generated in our previous study (OdaIshii et al., 2016), revealed a clear peak of Gata.a binding to the conserved upstream regions of each copy of Zic-r.b (Fig. 2A; Fig. $\mathrm{S} 2 \mathrm{~B})$. A lac $Z$ reporter construct containing this conserved upstream region was indeed activated in NN, aMM and pMM cells (Fig. 2B, C) (Anno et al., 2006).

In order to address whether the Gata sites were required for the expression driven by this conserved region, we introduced mutations in the Gata sites. We focused on the two proximal Gata sites for two reasons. First, the distal Gata sites are present in a region of the Zic-r. $b$ upstream regulatory sequences specifically required for expression in MM cells (Anno et al., 2006). Second, the two proximal Gata sites were conserved in the upstream sequence of Zic-r.b of the closely related Ciona savignyi. We introduced three different mutations into both of the two proximal Gata sites. In each case, reporter gene expression driven by these mutant constructs was greatly reduced in both NN and MM lineages (Fig. 2B-E; Fig. S3). The second mutant construct ( $\mu 2$; Fig. 2B,E) reveals that not only the core sequence, 'GATA', but also its flanking nucleotides are important, consistent with previous studies (Farley et al., 2015; Horikawa et al., 2013). Furthermore, gel-shift assays showed that the upstream region of Zic-r.b containing the proximal Gata sites (Fig. 2A; Fig. S2A) bound Gata.a protein in vitro, and that this binding was dependent upon intact Gata binding sites (Fig. 2F). Taken together, our results suggest that Gata.a directly activates Zic-r.b expression.

Curiously, although the Gata.a-MO analysis suggested that Gata.a activity is required for Zic-r.b expression in NN and aMM, but not pMM cells, our mutational analysis of the upstream regulatory sequences of Zic-r. $b$ suggests that the proximal Gata sites are required for activation in all the marginal cells (NN, aMM and $\mathrm{pMM})$ (Fig. 2B-E). This suggests that Gata.a contributes to Zic-r.b expression in $\mathrm{pMM}$ cells, but that transcription factors other than Gata.a are sufficient to control endogenous Zic-r.b expression in these cells. Regulatory elements that control this putative Gata.aindependent expression did not appear to be present even in a reporter construct containing longer upstream sequences (-682; Fig. S3).

\section{B-catenin/Tcf7 reduces the DNA-binding activity of Gata.a}

We next tested the possibility that $\beta$-catenin/Tcf7 prevents Gata.a from binding to the upstream regulatory sequences of Zic-r.b, as it does in the upstream regions of two ectoderm genes, Efna.d and Tfap2-r.b (Oda-Ishii et al., 2016). Consistent with this idea, gel-shift assays showed that Gata.a binding to the upstream sequences of $Z i c-r . b$ was greatly reduced when Gata.a protein was co-incubated with $\beta$-catenin and Tcf7 (Fig. 3A,B). Thus, the in vitro binding activity of Gata.a protein to the Gata sites of the Zic-r.b upstream region was negatively regulated by the presence of $\beta$-catenin and Tcf7.

We have previously shown that treatment of Ciona embryos with a pharmacological inhibitor of Gsk3 (BIO, GSK-3 inhibitor IX) stabilizes $\mathrm{n} \beta$-catenin and results in loss of Zic-r.b expression in NN and aMM cells (Hudson et al., 2013) (Fig. 3C,D). Based on the results presented so far, we predicted that in embryos treated with BIO, Gata.a would exhibit reduced binding to the upstream regulatory sequences of Zic-r.b. To test this, we performed a ChIP experiment with anti-Gata.a antibodies followed by quantitative 
A

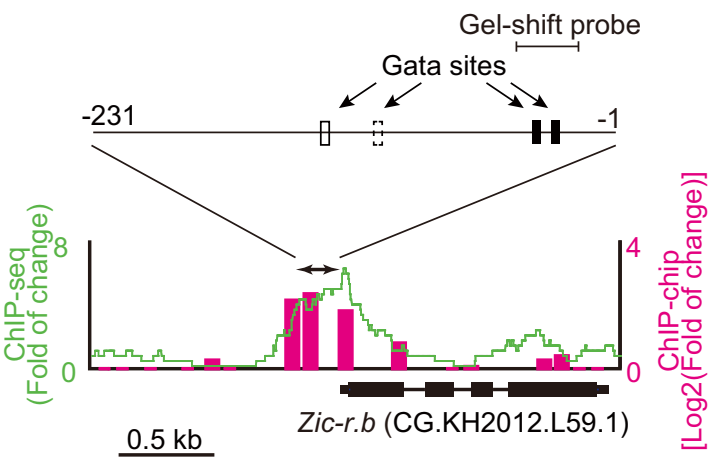

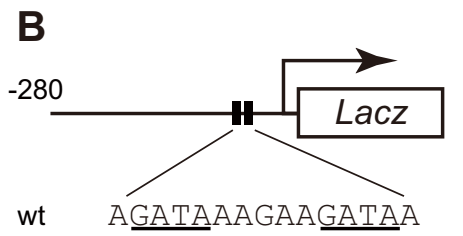

$\mu 1 \quad$ AattAAAgAA $\underline{\underline{a A t A A}}$

wt AGATAAAGAAGATAA

$\mu 2$

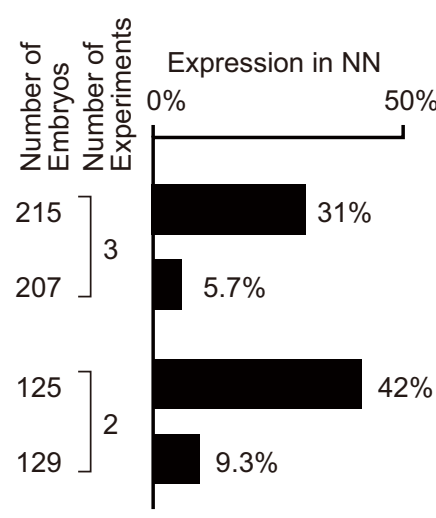

Expression in aMM

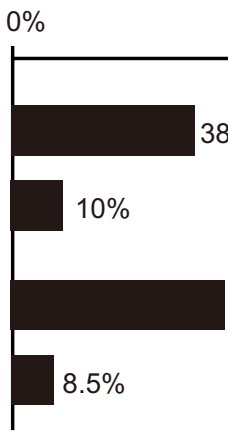

$43 \%$

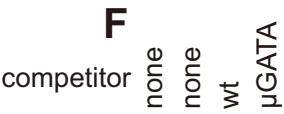

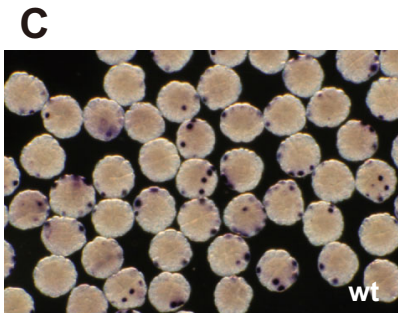

D

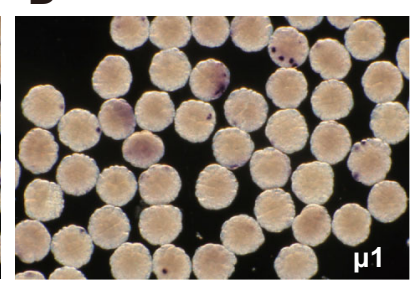

E

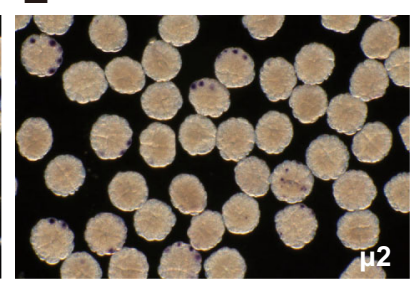

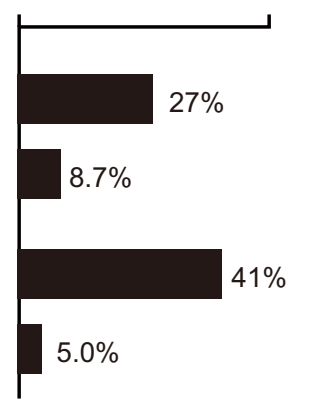

Expression in $\mathrm{pMM}$

50\%

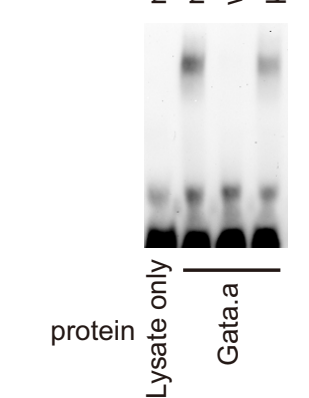

Fig. 2. Gata.a directly regulates Zic-r.b expression. (A) Mapping of Gata.a ChIP data, which were published previously (Oda-Ishii et al., 2016), onto a genomic region consisting of the exons and upstream region of Zic-r.b. The ChIP-chip data are shown in bars and the ChIP-seq data are shown as a green line. Each graph shows the fold enrichment ( $y$-axis) for the chromosomal region ( $x$-axis). A double-headed arrow indicates the upstream region that is highly conserved among four copies of Zic-r.b (Fig. S2A). This region contains four conserved Gata sites. The proximal Gata sites are shown by black boxes, and the distal Gata sites are shown by white boxes. The second site is not conserved in this copy, and is indicated with a dashed line. Mapping data onto genomic regions containing the other three copies are shown in Fig. S2B. (B-E) Analysis of a regulatory region of Zic-r.b. (B) The lacZ reporter constructs are depicted on the left with the two proximal Gata sites indicated by the black boxes. The sequences of intact (wt) and mutated $(\mu 1, \mu 2)$ Gata sites are shown below. The numbers indicate the relative nucleotide positions from the transcription start site of Zic-r.b, which was determined previously (Satou et al., 2006). The graph shows the percentage of NN, aMM and pMM cells that expressed lacZ mRNA at the 32-cell stage. Note that although there are four MM and four NN cells in a 32-cell stage embryo, not all cells or embryos will express the reporter because of mosaic incorporation of the electroporated constructs. (C-E) Photographs showing expression of lacZ mRNA (detected by in situ hybridization) in embryos electroporated with wt (C), $\mu 1$ (D) and $\mu 2$ (E) constructs. (F) Gel-shift analysis showing that the proximal Gata sites in the upstream region of Zic-r.b bound Gata.a protein in vitro. The shifted band disappeared by incubation with a specific competitor (wt), but not the competitor with mutant Gata.a-binding sites ( $\mu$ GATA). The mutated sequence is the same as the one shown in Fig. S3.

PCR (Fig. 3E). In all three independent experiments, Gata.a binding to the upstream region of Zic-r. $b$ was reduced in embryos cultured in BIO. We conclude that $\beta$-catenin/Tcf activity reduces in vivo binding of Gata.a to the target sites in the Zic-r.b upstream region.

\section{Conclusions}

Our results show that antagonism between Gata.a and $\beta$-catenin/ Tcf7 are central to both rounds of $\beta$-catenin-dependent switches that segregate the germ layers during early ascidian embryogenesis
(Fig. 4). This antagonistic relationship first segregates the ectodermal lineages (Gata.a dependent) from the endomesodermal lineages (n $\beta$-catenin dependent) at the 16-cell stage, and then the mesodermal/neural lineages (Gata.a dependent) from the endodermal lineages (n $\beta$-catenin dependent) at the 32-cell stage. Thus, the two $\mathrm{n} \beta$-catenin/Gata.a switches at the 16- and 32-cell stages subdivide the Ciona embryo into three broad domains that correspond to the segregating germ layers. The second $n \beta$-catenin/ Gata.a switch cooperates with the lineage-specific factors inherited 
A

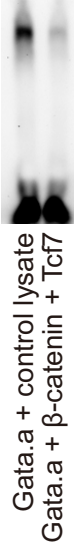

E

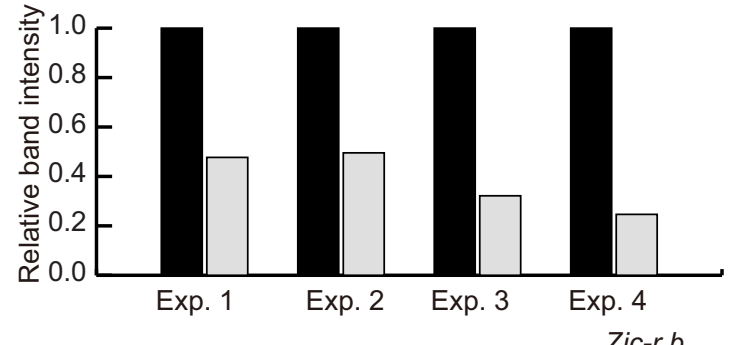

C
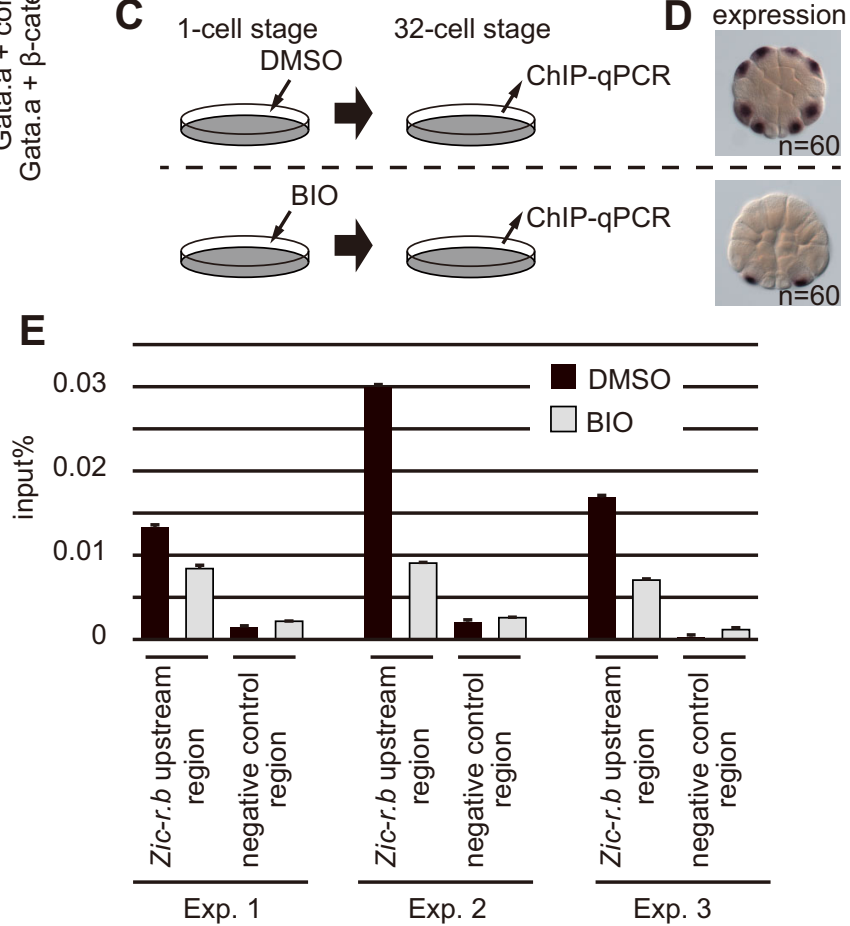

Fig. 3. Gata.a binding activity is reduced by interaction with Tcf7 and $\beta$-catenin. (A) Gel-shift analysis showing suppression of Gata.a binding activity by Tcf7 and $\beta$-catenin. Whereas Gata.a protein produced in vitro bound to the upstream sequences containing the Gata.a sites (left lane), coincubation of Gata.a with $\beta$-catenin and Tcf7 reduced this binding (right lane). In the left lane, the same amount of a rabbit reticulocyte lysate was added for control instead of $\beta$-catenin and Tcf7. (B) Quantification of the shifted bands in four independent gel-shift experiments. (C-E) ChIP followed by quantitative $\mathrm{PCR}$ showing that BIO treatment of embryos reduces Gata-a binding to the Zic-r.b upstream region. (C) The experimental design. BIO treatment ectopically stabilizes $\beta$-catenin. (D) BIO treatment from the one-cell stage abolished Zic-r.b expression in NN and aMM cells, as does BIO treatment from the eight-cell or 16-cell stage (Hudson et al., 2013). (E) Three biological replicates indicate that $\mathrm{BIO}$ treatment reduced Gata.a binding to the Zic-r.b upstream region. Error bars indicate s.d. among qPCR technical replicates.

from the endomesoderm precursors, during activation of the mesoderm- and endoderm-specific target genes (Fig. 4). Foxd, activated by the first $n \beta$-catenin/Gata.a switch in the endomesoderm precursors of the 16-cell embryo (Imai et al., 2002a), is inherited by the both daughter cells of the 32-cell embryo (Hudson et al., 2013) where it co-operates with the second $n \beta$-catenin/Gata.a switch to activate distinct gene expression. Consistent with this idea, previous studies have shown that Foxd is required for activation of Zic-r.b (Imai et al., 2002b), and can bind directly to its upstream sequences (Kubo et al., 2010). Indeed, there are four putative Fox binding sites in the upstream regions (Fig. S2A) (Anno et al., 2006), and 16-cell stage
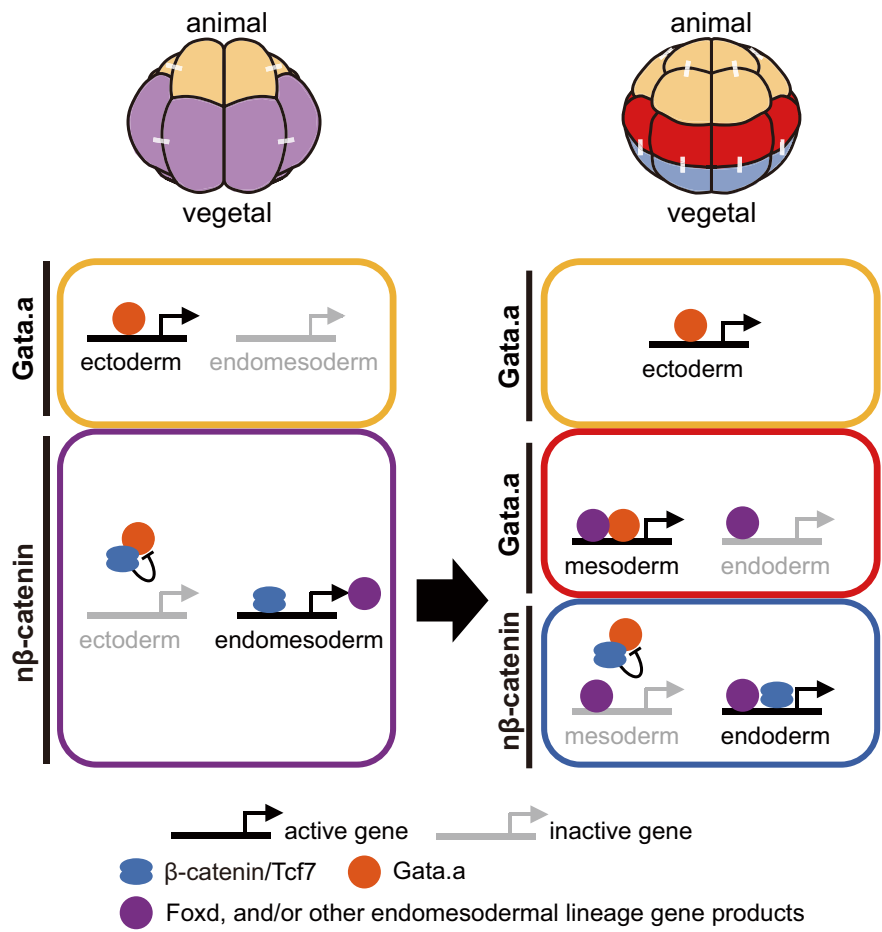

Fig. 4. Antagonistic action of Gata.a and $\beta$-catenin/Tcf7 during early germ layer segregation. The diagrams at the top represent an anterior (notochord side) view of 16- and 32-cell embryos. In 16-cell embryos, $\beta$-catenin is localized to nuclei of the vegetal hemisphere (purple) (Hudson et al., 2013; Imai et al., 2000) where it activates 'endomesoderm' target genes such as Foxd (Imai et al., 2002a), and suppresses Gata.a activity by physical interaction (Oda-Ishii et al., 2016). In the animal hemisphere (yellow), Gata.a is free from suppression by $\beta$-catenin/Tcf7, and activates its 'ectoderm' target genes, which include Efna.d and Tfap2-r.b (Oda-Ishii et al., 2016; Rothbächer et al., 2007). In 32-cell embryos, $n \beta$-catenin is activated in $E$ cells (blue) but not margin cells (red). In E cells, $\beta$-catenin/Tcf7, together with the endomesoderm gene products, activates 'endoderm' genes, which include Lhx3/4 (Hudson et al., 2013; Imai et al., 2006), and suppresses Gata.a activity. In the margin, Gata.a is now free from $\beta$-catenin/ Tcf7-mediated inhibition and, together with 'endomesoderm' gene products, it activates 'mesoderm' genes such as Zic-r.b.

mutations introduced into these Fox sites abolished reporter gene expression in NN and aMM cells (Fig. S4A-C), matching the required role of Foxd for Zic-r.b expression (Fig. S4D). Similarly, Foxa.a is activated in the endomesoderm precursors of the 16-cell embryo (Imai et al., 2004), and is required for correct Zic-r.b expression in NN and aMM cells (Fig. S4E) (Hudson et al., 2016).

The mechanism of $n \beta$-catenin-mediated transcriptional repression described in the present and previous (Oda-Ishii et al., 2016) studies is different from other mechanisms described in Caenorhabditis elegans and Drosophila melanogaster (Bertrand, 2016; Blauwkamp et al., 2008; Murgan et al., 2015). Thus, nuclear $\beta$-catenin-dependent transcriptional repression appears to be mediated by diverse mechanisms.

\section{MATERIALS AND METHODS}

\section{Animals}

C. intestinalis adults were obtained from the National Bio-Resource Project for Ciona in Japan or purchased from the Station Biologique de Roscoff in France.

\section{In situ hybridization}

Whole-mount in situ hybridization was performed as described previously (Hudson et al., 2013; Imai et al., 2004). Identifiers for genes examined in the 
present study were as follows (Satou et al., 2008; Stolfi et al., 2015): CG. KH2012.C8.396/CG.KH2012.C8.890 for Foxd, CG.KH2012.L20.1 for Gata.a, CG.KH2012.C9.53 for $\beta$-catenin, CG.KH2012.C6.71 for Tcf7, and CG.KH2012.L59.12/CG.KH2012.L59.1/CG.KH2012.S816.1/CG.

KH2012.S816.4 for Zic-r.b.

\section{Knockdown studies}

The MO (Gene Tools, LLC) against Gata.a, which blocks translation, has been used previously (5'-GGGTTAGGCATATACATTCTTTGGA-3') (Bertrand et al., 2003; Oda-Ishii et al., 2016; Rothbächer et al., 2007). A second MO that differs by two nucleotides was also used (5'-GGTTAGGCATATACATTCTTTGGAA-3') and gave similar results (Fig. 1D). We also used a MO against $E$. coli lac $Z$ as a negative control (5'-TACGCTTCTTCTTTGGAGCAGTCAT- $3^{\prime}$ ). These MOs were introduced by microinjection under a stereo-microscope. The conserved upstream region present in each of our reporter assay constructs is depicted in Fig. S2A. Reporter constructs were introduced into fertilized eggs by electroporation (Corbo et al., 1997). All knockdown experiments and reporter assays were performed at least twice independently.

\section{ChIP experiments}

Mapping of our previously published ChIP-chip (GEO accession number GSE70902) and ChIP-seq (SRA accession number: DRA003742) data was performed as described previously (Oda-Ishii et al., 2016). Gel-shift assays were also performed as described previously (Oda-Ishii et al., 2016), using a digoxigenin-labeled probe and Gata.a protein synthesized with a rabbit reticulocyte lysate system (Promega). The sequence of the probe is shown in Fig. S2A. Chromatin-immunoprecipitation followed by quantitative PCR was performed as described previously (Oda-Ishii et al., 2016). The negative control primer set was the same as that used in a previous study (Oda-Ishii et al., 2016). The primer sequences to amplify the upstream sequence of Zic$r . b$ are shown in Fig. S2A. BIO (Merck Millipore) was used at $2.5 \mu \mathrm{M}$

\section{Acknowledgements}

We thank the National Bio-resource project (MEXT, Japan) and the Roscoff Marine Station (France) for providing experimental animals.

\section{Competing interests}

The authors declare no competing or financial interests.

\section{Author contributions}

K.S.I, C.H., I.O., H.Y. and Y.S. performed the experiments. C.H. and Y.S. wrote the paper. K.S.I., H.Y. and Y.S. conceived the project.

\section{Funding}

This research was supported by Grants-in-Aid from the Japanese Ministry of Education, Culture, Sports, Science, and Technology; the Japan Society for the Promotion of Science [26711014 to K.S.I., 25118510 to Y.S.]; a CREST program of Japan Science and Technology Agency (JST) (Y.S.); the Centre National de la Recherche Scientifique (CNRS); the Université Pierre et Marie Curie; the Fondation ARC pour la Recherche sur le Cancer [1144 and PJA 20131200223]; and the Agence Nationale de la Recherche [ANR-09-BLAN-0013-01 to H.Y.]

\section{Supplementary information}

Supplementary information available online at

http://dev.biologists.org/lookup/doi/10.1242/dev.141481.supplemental

\section{References}

Anno, C., Satou, A. and Fujiwara, S. (2006). Transcriptional regulation of ZicL in the Ciona intestinalis embryo. Dev. Genes Evol. 216, 597-605.

Bertrand, V. (2016). beta-catenin-driven binary cell fate decisions in animal development. Wiley Interdiscipl. Rev. Dev. Biol. 5, 377-388.

Bertrand, V., Hudson, C., Caillol, D., Popovici, C. and Lemaire, P. (2003). Neural tissue in ascidian embryos is induced by FGF9/16/20, acting via a combination of maternal GATA and Ets transcription factors. Cell 115, 615-627.

Blauwkamp, T. A., Chang, M. V. and Cadigan, K. M. (2008). Novel TCF-binding sites specify transcriptional repression by Wnt signalling. EMBO J. 27 1436-1446.

Corbo, J. C., Levine, M. and Zeller, R. W. (1997). Characterization of a notochordspecific enhancer from the Brachyury promoter region of the ascidian, Ciona intestinalis. Development 124, 589-602.
Darras, S., Gerhart, J., Terasaki, M., Kirschner, M. and Lowe, C. J. (2011). betaCatenin specifies the endomesoderm and defines the posterior organizer of the hemichordate Saccoglossus kowalevskii. Development 138, 959-970.

Dehal, P., Satou, Y., Campbell, R. K., Chapman, J., Degnan, B., De Tomaso, A., Davidson, B., Di Gregorio, A., Gelpke, M., Goodstein, D. M. et al. (2002). The draft genome of Ciona intestinalis: insights into chordate and vertebrate origins. Science 298, 2157-2167.

Farley, E. K., Olson, K. M., Zhang, W., Brandt, A. J., Rokhsar, D. S. and Levine, M. S. (2015). Suboptimization of developmental enhancers. Science 350 325-328.

Horikawa, Y., Matsumoto, H., Yamaguchi, F., Ishida, S. and Fujiwara, S. (2013) Transcriptional regulation in the early ectodermal lineage of ascidian embryos. Dev. Growth Differ. 55, 776-785

Hudson, C., Kawai, N., Negishi, T. and Yasuo, H. (2013). B-catenin-driven binary fate specification segregates germ layers in ascidian embryos. Curr. Biol. 23 , 491-495.

Hudson, C., Sirour, C. and Yasuo, H. (2016). Co-expression of Foxa.a, Foxd and Fgf9/16/20 defines a transient mesendoderm regulatory state in ascidian embryos. Elife 5, e14692.

Imai, K., Takada, N., Satoh, N. and Satou, Y. (2000). $\beta$-catenin mediates the specification of endoderm cells in ascidian embryos. Development 127 3009-3020.

Imai, K. S., Satoh, N. and Satou, Y. (2002a). An essential role of a FoxD gene in notochord induction in Ciona embryos. Development 129, 3441-3453.

Imai, K. S., Satou, Y. and Satoh, N. (2002b). Multiple functions of a Zic-like gene in the differentiation of notochord, central nervous system and muscle in Ciona savignyi embryos. Development 129, 2723-2732.

Imai, K. S., Hino, K., Yagi, K., Satoh, N. and Satou, Y. (2004). Gene expression profiles of transcription factors and signaling molecules in the ascidian embryo: towards a comprehensive understanding of gene networks. Development 131 4047-4058.

Imai, K. S., Levine, M., Satoh, N. and Satou, Y. (2006). Regulatory blueprint for a chordate embryo. Science 312, 1183-1187.

Kubo, A., Suzuki, N., Yuan, X., Nakai, K., Satoh, N., Imai, K. S. and Satou, Y. (2010). Genomic cis-regulatory networks in the early Ciona intestinalis embryo. Development 137, 1613-1623.

Logan, C. Y., Miller, J. R., Ferkowicz, M. J. and McClay, D. R. (1999). Nuclear $\beta$-catenin is required to specify vegetal cell fates in the sea urchin embryo. Development 126, 345-357.

Maduro, M. F. (2009). Structure and evolution of the C. elegans embryonic endomesoderm network. Biochim. Biophys. Acta Gene Regul. Mech. 1789 , 250-260.

McCauley, B. S., Akyar, E., Saad, H. R. and Hinman, V. F. (2015). Dosedependent nuclear beta-catenin response segregates endomesoderm along the sea star primary axis. Development 142, 207-217.

Momose, T. and Houliston, E. (2007). Two oppositely localised frizzled RNAs as axis determinants in a Cnidarian embryo. PLoS Biol. 5, e70.

Murgan, S., Kari, W., Rothbacher, U., Iche-Torres, M., Melenec, P., Hobert, O. and Bertrand, V. (2015). Atypical transcriptional activation by TCF via a Zic transcription factor in C. elegans neuronal precursors. Dev. Cell 33, 737-745.

Oda-Ishii, I., Kubo, A., Kari, W., Suzuki, N., Rothbacher, U. and Satou, Y. (2016). A maternal system initiating the zygotic developmental program through combinatorial repression in the Ascidian embryo. PLoS Genet. 12, e1006045.

Rothbächer, U., Bertrand, V., Lamy, C. and Lemaire, P. (2007). A combinatoria code of maternal GATA, Ets and $\beta$-catenin-TCF transcription factors specifies and patterns the early ascidian ectoderm. Development 134, 4023-4032.

Satou, Y. and Imai, K. S. (2015). Gene regulatory systems that control gene expression in the Ciona embryo. Proc. Jpn. Acad. Ser. B Physiol. Biol. Sci. 91 33-51.

Satou, Y., Imai, K. S. and Satoh, N. (2001). Early embryonic expression of a LIMhomeobox gene $\mathrm{Cs}-\mathrm{lh} \times 3$ is downstream of beta-catenin and responsible for the endoderm differentiation in Ciona savignyi embryos. Development 128 3559-3570.

Satou, Y., Hamaguchi, M., Takeuchi, K., Hastings, K. E. M. and Satoh, N. (2006). Genomic overview of mRNA 5 '-leader trans-splicing in the ascidian Ciona intestinalis. Nucleic Acids Res. 34, 3378-3388.

Satou, Y., Mineta, K., Ogasawara, M., Sasakura, Y., Shoguchi, E., Ueno, K. Yamada, L., Matsumoto, J., Wasserscheid, J., Dewar, K. et al. (2008). Improved genome assembly and evidence-based global gene model set for the chordate Ciona intestinalis: new insight into intron and operon populations. Genome Biol. 9, R152.

Stolfi, A., Sasakura, Y., Chalopin, D., Satou, Y., Christiaen, L., Dantec, C., Endo, T., Naville, M., Nishida, H., Swalla, B. J. et al. (2015). Guidelines for the nomenclature of genetic elements in tunicate genomes. Genesis 53, 1-14.

Wikramanayake, A. H., Huang, L. and Klein, W. H. (1998). beta-Catenin is essential for patterning the maternally specified animal-vegetal axis in the sea urchin embryo. Proc. Natl. Acad. Sci. USA 95, 9343-9348.

Wikramanayake, A. H., Hong, M., Lee, P. N., Pang, K., Byrum, C. A., Bince, J. M., Xu, R. H. and Martindale, M. Q. (2003). An ancient role for nuclear beta-catenin in the evolution of axial polarity and germ layer segregation. Nature 426, 446-450. 
Yagi, K., Satou, Y. and Satoh, N. (2004). A zinc finger transcription factor, ZicL, is a direct activator of Brachyury in the notochord specification of Ciona intestinalis. Development 131, 1279-1288.
Yamada, L., Kobayashi, K., Degnan, B., Satoh, N. and Satou, Y. (2003). A genomewide survey of developmentally relevant genes in Ciona intestinalis. IV. Genes for HMG transcriptional regulators, bZip and GATA/Gli/Zic/Snail. Dev. Genes Evol. 213, 245-253. 